Results According to the results of LIQT: normolactasia (NL) was observed in 26\%, moderate hypolactasia (GL) - in 35\% and severe GL - in 39\% patients with Crohn's disease. In children with Ulcerative colitis NL was observed in $31,5 \%$, moderate GL - in 32,5\% and severe GL - in 36\%. In the patients with normolactasia we revealed an increase in the number of genotypes $T T$ of the of the polymorphic marker rs182549 (c.13910 C>T). In patients with moderate and severe GL, the genotype CC of this polymorphic marker was significantly more frequent $(\mathrm{P}=0.030 ; \mathrm{OR}=2.72 ; 95 \% \mathrm{CI}$, 1.08-6.87).

Dairy products «open food challenge» was performed in 20 children with IBD and suspected CMA (questionnaire score 54-71). CMA was confirmed in 12 patients by the results of the morphological study of intestinal biopsy specimens with the counting of eosinophils and «open food challenge». According to these findings diet recommendation for patient with IBD were personalized: Cow's milk-free diet for children with CMA, Lactose-free diet - for children with severe lactose intolerance, Low lactose diet was recommended to the patients with moderate hypolactasia, Diet containing Cow's milk - for other patients. Children with GL and genotypes TT had better prognosis for lactosetolerance.

Conclusion Allergy-focused history questionnaire for identifying of CMA, Lactose Intolerance quick test and assessment of genotype of polymorphic marker rs182549 in the MCM6 gene may improve approaches for personalization of diet for patients with IBD.

\section{GP175 MUCOSAL ATROPHY AND CLINICAL CORRELATION IN CHILDREN WITH ULCERATIVE COLITIS}

Lorraine Stallard*, Seamus Hussey. Department of Gastroenterology, Our Lady's Children's Hospital Crumlin, Dublin, Ireland

\subsection{6/archdischild-2019-epa.236}

Background Mucosal healing is now preferred over clinical remission as a target endpoint in the treatment of Ulcerative Colitis (UC) as it has been demonstrated to reduce hospitalisation and corticosteroid use, to reduce the risk of colectomy and to reduce the risk of colorectal cancer. In routine practice, the activity and severity of UC is based on combined clinical and endoscopic assessment in addition to histology. However, it is not clear whether the currently available histologic indices can help predict disease course and treatments. Mucosal atrophy (MA) describes a state of extensive mucosal architectural damage, causing irregularities in the morphology and distribution of the intestinal glands of the mucosa. The aim of this study was to investigate the prevalence of MA in a paediatric cohort of newly diagnosed patients with UC and evaluate the relationship between the presence of MA, disease activity and treatments.

Methods Children $<17$ years old diagnosed with UC and recruited to the DOCHAS study (Determinants and Outcomes in Children and Adolescents with IBD) between January 2012 and December 2016 were included in the study. Colonic biopsies were evaluated for the presence of MA at time of diagnosis in treatment naïve patients with UC. Correlation with clinical indices was performed using the Paediatric Ulcerative Colitis Activity Index (PUCAI). Follow up data including
PUCAI, current treatment and relapse rates were collected at 3 months, 6 months, 12 months and yearly thereafter.

Results 176 patients with UC were included of which 21 (12\%) had MA on biopsy at diagnosis (mean age 11.9 years, $51 \%$ female). Those with MA had higher PUCAI scores at three month $(p=0.04)$, six month $(p=0.01)$ and two year $(\mathrm{p}=0.01)$ follow up. MA patients had significantly higher rates of progression to immunomodulators $(58 \%$ vs $44 \%)$ and biologics $(25 \%$ vs $11 \%)$, were more likely to have a clinical relapse $(p=0.04)$ and had shorter times to first relapse post diagnosis $(p=0.01)$.

Conclusion Children with UC and mucosal atrophy demonstrate a more severe disease course with higher disease activity scores and rates of relapse, which results in increased rates of progression to more intensive therapies. Further research into the mechanisms underlying the more severe disease course of mucosal atrophy patients is warranted.

\section{GP176 MORPHOLOGICAL FEATURES OF CHRONIC GASTRITIS ASSOCIATED WITH EPSTEIN-BARR VIRUS IN CHILDREN}

${ }^{1}$ Valeria Novikova*, ${ }^{1}$ Olga Gurina, ${ }^{1}$ Olga Varlamova, ${ }^{1}$ Aleksey Blinov, JeanClaude Hakizimana ${ }^{1},{ }^{1}$ Yulia Karpeeva, ${ }^{2}$ Andrey Petrovsky. ${ }^{1}$ St. Petersburg State Pediatric Medical University, Saint Petersburg, Russian Federation; ${ }^{2}$ North-Western State Medical University named after I.I. Mechnikov, Saint Petersburg, Russian Federation

\subsection{6/archdischild-2019-epa.237}

Introduction The Epstein-Barr virus has been recently considered as an etiological cause of chronic gastritis.

Objectives To study the relationship between the morphological features of chronic gastritis and Epstein-Barr virus infection.

Methods 50 children (mean age $12.4 \pm 0.3$ years) with morphologically verified chronic gastritis were examined. In All patients, fibrogastroduodenoscopy was carried out and two biopsy samples from the body and antrum parts of the stomach were taken. Morphological assessment of biopsy specimens was done according to a standard visual analogue scale. The EBV was determined by immunohistochemical method. The EBV antibodies anti-EA IgG and anti-EBNA IgM were determined by ELISA using standard sets manufactured by 'Vector Best' company. Statistical processing of research materials was carried out using software and package Statistica for Windows (version 6.0).

Results Anti-EA IgG to EBV, indicating active virus replication, were identified in 36 children (72\%). Antibodies to EBV class IgG $\mathrm{M}$, characteristic of the acute stage of EBV infection, had not been found in any child. EBV was detected in $71.4 \%$ of biopsy specimens from the stomach of the children who had been tested anti-EA IgG positive. The presence of EBV antigens in the gastric epithelium revealed significant correlation with the presence of hemorrhages in the body of the stomach $(\mathrm{r}=0.68 ; \mathrm{p}<0.05)$ and in the antrum $(\mathrm{r}=0.66 ; \mathrm{p}<0.05)$, microthrombosis in the body of the stomach $(\mathrm{r}=0.38 ; \mathrm{p}<0.05)$ and in the antrum $(r=0.42 ; \mathrm{p}<0.05)$, regenerative changes in the cervical epithelium of the gastric mucosa $(r=0.32 ; p<0.05)$, fibrosis in the mucosa of the body of the stomach $(r=0.46$; $\mathrm{p}<0.05$ ), atrophy of the glands of the body of the stomach $(\mathrm{r}=0.65 ; \mathrm{p}<0.05)$, atrophy of antral glands $(\mathrm{r}=0.52 ; \mathrm{p}<0.05)$, pylorization of fundal glands $(\mathrm{r}=0.5 ; \mathrm{p}<0.05)$, hyperplasia of parietal cells $(r=0.62 ; \mathrm{p}<0.05)$, erosion in the body of the stomach $(\mathrm{r}=0.47 ; \mathrm{p}<0.05)$, erosion in the antrum $(\mathrm{r}=0.36$; $\mathrm{p}<0.05)$. 
Conclusions Studies showed that in children with chronic gastritis, Epstein-Barr virus markers are detected in the blood with high frequency. There is a relationship between the presence of the Epstein-Barr virus in the gastric mucosa and histological signs of gastritis.

\section{GP177 ASSESSMENT OF NUTRITIONAL AND IRON STATUS OF HELICOBACTER PYLORI INFECTED CHILDREN - A SINGLE CENTER STUDY}

${ }^{1}$ Luiza Bordei, 'Victoria Hurduc, ${ }^{1}$ Doina Anca Plesca*. 'Victor Gomoiu Clinical Children's Hospital, 'Carol Davila', University Of Medicine and Pharmacy, Bucharest, Romania; ${ }^{2}$ Victor Gomoiu Clinical Children's Hospital, 'Carol Davila', University Of Medicine and Pharmacy, Bucharest, Romania

10.1136/archdischild-2019-epa.238

Introduction Helicobacter pylori ( $H$ pylori) infection affects about $30 \%$ to two-thirds of human populations and is usually acquired in early childhood. There are conflicting results regarding the nutritional effects of $H$ pylori infection in children mostly about the reduced bioavailability of essential nutrients with growth impairment.

The association between $\mathrm{H}$ pylori infection and iron deficiency anaemia is of considerable current interest.

Objectives To evaluate the effects of $H$ pylori infection on the nutritional and the iron status of symptomatic children that required a first upper endoscopic evaluation.

Methods This was an observational prospective study of 649 symptomatic children (age range 6 months-18 years) mostly with uninvestigated dyspepsia or extradigestive signs, admitted in our unit, from January 2015 to December 2017. Weight, height, body mass index for age and sex were used according to growth charts provided by WHO, 2007. Romania is in a nutritional transition and does not have updated national growth charts. $\mathrm{H}$ pylori infection was documented by at least two standard invasive tests. Hematologic parameters and nutritional status were compared in patients with and without $H$ pylori infection.

Results Active $H$ pylori infection was documented in mostly of studied patients $(63,64 \%)$. The majority of patients presented normal nutritional status $(67,32 \%)$, with a significant proportion of wasting $(12,63 \%)$ associated with risk to underweight $(13,09 \%)$ overweight $(4,48 \%)$ and obesity $(2.16 \%)$. The prevalence of undernutrition was higher in uninfected $\mathrm{H}$ pylori children compared with the infected ones $(13,55 \%$ versus $12,1 \% ; p=0,67)$. Overnutrition prevalence was higher in the case of the $\mathrm{H}$ pylori negative children compared to the positive ones $(3,81 \%$ versus $2,66 \%, p=0,37)$. The stunted was observed only in $3.08 \%$ cases. Iron deficiency anaemia was found in $19,72 \%$, with an approximately the same prevalence in uninfected compared to infected children $(19,91 \%$ versus 19,61\%).

Conclusions The $H$ pylori prevalence rate $(63,64 \%)$ revealed by our study suggests that this infection remains a semnificative problem in our country. This endoscopic series revealed a lower prevalence of undernutrition and overnutrition in symptomatic $H$ pylori infected children compared with uninfected ones, but without statistically signification for the both ends of the spectrum of poor nutritional status. Our study showed that the prevalence of iron deficiency anaemia was not significantly higher in uninfected $\mathrm{H}$ pylori children compared to infected pacients. According to other observational studies the role and the impact of $\mathrm{H}$ pylori infection on growth and iron deficiency anaemia remains controversial.

\section{GP178 MORPHOLOGICAL FEATURES OF CHRONIC ESOPHAGITIS IN CHILDREN WITH CONCOMITANT ALLERGIC DISEASES ARE INTERRELATED WITH THE LEVEL OF CYTOKINES AND NEUROPEPTIDES}

${ }^{1}$ valeria novikova*, ${ }^{2}$ Nikolay Anichkov, ${ }^{2}$ Elena Nevskaia, ${ }^{1}$ Anastasia Listopadova, ${ }^{1,2}$ Yulia Zamiatina, ${ }^{1}$ Olga Gurina, ${ }^{1}$ Olga Varlamova, ${ }^{1}$ Aleksey Blinov. ${ }^{1}$ St. Petersburg State Pediatric Medical University, Saint Petersburg, Russian Federation; ${ }^{2}$ North-Western State Medical University named after I.I. Mechnikov, Saint Petersburg, Russian Federation

10.1136/archdischild-2019-epa.239

Introduction IgE-mediated as well as non-IgE-mediated inflammation to the mucous membranes of the gastrointestinal tract is possible in children with allergic diseases. Purpose of the study: We investigated the morphological features of chronic esophagitis in children with atopic dermatitis (AtD) and bronchial asthma (BA) taking in account the cytokine status (IL-4, IL-5 and IL-13), the level of markers of eosinophilic inflammation (eosinophilic cationic ECP protein and eosinophilic neurotoxin EDN) and neuropeptides (neuropeptide Y (NPY) and substance $\mathrm{P}(\mathrm{SP})$ ).

Patients and methods Study included 61 children (mean age $13.9 \pm 2.36$ years) having morphologically verified chronic esophagitis. 20 children suffered from concomitant AtD, 21 had esophagitis with BA. 20 children had esophagitis without concomitant atopic diseases. All children have undergone esophagogastroduodenoscopy and sampling of mucosal biopsy material from the middle third of the esophagus. Histologically we evaluated dystrophic, regenerative, inflammatory changes, vascular disorders and defects of the mucous membrane. In all children, the level of total IgE was determined and a blood test for IL-4, IL-5 and IL-13, ECP, EDN, NPY and SP was performed by ELISA. The statistical results were estimated by use of the program Stat Soft Statistica 12.0. for Windows- 10.

Research results Morphological features of esophagitis in all studied groups equally frequent. The average level of total $\mathrm{IgE}$ in children with AtD and esophagitis was 186.6 $\pm 126.6 \mathrm{U} / \mathrm{ml}$; in the group of children with BA was $331,5 \pm 238,6 \mathrm{U} / \mathrm{ml}$. All children with esophagitis without atopic diseases had a level of total IgE within the normal range. In AtD, IL-4 levels $(1.08 \pm 0.47 \mathrm{pg} / \mathrm{ml}$ and $1.91 \pm 0.88 \mathrm{pg} / \mathrm{ml}, \mathrm{p}<0.05)$, IL-5 (21.39 $\pm 15.47 \mathrm{pg} / \mathrm{ml}$ and $1.91 \pm 0.88 \mathrm{pg} / \mathrm{ml}, \mathrm{p}<0.05)$, IL-13 (1.72 $\pm 1.55 \mathrm{pg} /$ $\mathrm{ml}$ and $12.11 \pm 6.10 \mathrm{pg} / \mathrm{ml}, \mathrm{p}<0.05), \mathrm{EDN}(47.70 \pm$ $34.83 \mathrm{pg} / \mathrm{ml}$ and $119.00 \pm 64.26 \mathrm{pg} / \mathrm{ml}, \mathrm{p}<0.05)$ and NPY $(40.62 \pm 20.55 \mathrm{pg} / \mathrm{ml}$ and $160.44 \pm 144.44 \mathrm{pg} / \mathrm{ml}$, $\mathrm{p}<0.005$ ) were lower than with the combination of esophagitis with BA and had no differences with the group of children without atopy. Correlative relationship between morphological and immunological parameters in patients with atopy was found.

Conclusion Esophagitis in children with bronchial asthma is accompanied by higher levels of IgE and more pronounced changes in cytokine status than in atopic dermatitis. The correlative relationships between morphological and 